\title{
Linear free resolutions over non-commutative algebras
}

\author{
Peter Jørgensen
}

\begin{abstract}
The main result of this paper is that, over a non-commutative Koszul algebra, high truncations of finitely generated graded modules have linear free resolutions.
\end{abstract}

\section{Introduction}

Eisenbud and Goto [EG84], Avramov and Eisenbud [AE92], and the present author [Jor99b] have all studied whether high truncations of finitely generated graded modules over graded algebras have linear free resolutions.

The original study of this took place over polynomial algebras. The main result [EG84, Proposition, p. 89] is: If $M$ is a finitely generated graded module over $k\left[X_{1}, \ldots, X_{t}\right]$ with $k$ a field, then for large $s$, the minimal free resolution of the degree shifted truncation $M_{\geqslant s}(s)$ is linear. That is, the $m$ th module in the minimal free resolution has all its generators placed in degree $m$.

This was later extended in [AE92, Corollary 2] to commutative Koszul algebras, and in [Jor99b, Theorem 2.6] to non-commutative AS-regular algebras, which are algebras with good homological behaviour generalizing that of polynomial algebras.

This paper proves a common extension of [AE92, Corollary 2] and [Jor99b, Theorem 2.6]: If $A$ is a non-commutative Koszul algebra satisfying a few weak conditions given in Setup 0.1, then for any finitely generated graded module $M$ and large $s$, the minimal free resolution of $M_{\geqslant s}(s)$ is linear. This is Theorem 3.1 below.

Along the way, I prove Theorems 2.5 and 2.6 and Corollaries 2.8 and 2.9, which show that the two competing definitions of Castelnuovo-Mumford regularity of graded modules given in [AE92], respectively [EG84] and [Jor99b], are in fact closely related.

SETup 0.1. Throughout the paper, $k$ is a field, and $A$ is a noetherian $\mathbb{N}$-graded connected $k$-algebra which has a balanced dualizing complex.

See [Jor99a] and [Jor99b] for generalities on the theory of graded algebras, and [vdB97] and [Yek92] for information on dualizing complexes. My notation is mostly standard, but I do want to list a few items of terminology.

The opposite algebra of $A$ is denoted $A^{\text {opp }}$, and $A$-right-modules are identified with $A^{\text {opp }}$-leftmodules.

The abelian category of graded $A$-left-modules and graded homomorphisms of degree zero is denoted $\operatorname{Gr} A$. The derived category of $\operatorname{Gr} A$ is denoted $\mathrm{D}(\mathrm{Gr} A)$. If $X$ is in $\mathrm{D}(\mathrm{Gr} A)$, then $\mathrm{h}^{m} X$ denotes the $m$ th cohomology module of $X$. The derived category $\mathrm{D}(\mathrm{Gr} A)$ has full subcategories $\mathrm{D}^{-}(\mathrm{Gr} A)$ consisting of complexes $X$ with $\mathrm{h}^{m} X=0$ for $m$ large positive, $\mathrm{D}^{+}(\mathrm{Gr} A)$ consisting of complexes $X$ with $\mathrm{h}^{m} X=0$ for $m$ large negative, and $\mathrm{D}_{\mathrm{fg}}^{\mathrm{b}}(\mathrm{Gr} A)$ consisting of complexes $X$ with $\mathrm{h}^{m} X=0$ for $m$ large positive or negative and each $\mathrm{h}^{m} X$ finitely generated.

Received 17 February 2002, accepted in final form 17 September 2002.

2000 Mathematics Subject Classification 16E05, 16E30, 16W50.

Keywords: Castelnuovo-Mumford regularity, non-commutative Koszul algebra, linear free resolution.

This journal is (C) Foundation Compositio Mathematica 2004. 


\section{P. JøRGENSEN}

The derived functors of $\operatorname{Hom}_{A}$ and $\otimes_{A}$ are denoted $\mathrm{RHom} A$ and $\otimes_{A}$. Section functors of graded $A$-left- and graded $A$-right-modules are denoted $\Gamma_{\mathfrak{m}}$ and $\Gamma_{\mathfrak{m}}$ opp , and their derived functors are denoted $R \Gamma_{\mathfrak{m}}$ and $R \Gamma_{\mathfrak{m}^{\text {opp }}}$. These give rise to local cohomology functors $H_{\mathfrak{m}}^{m}=\mathrm{h}^{m} \mathrm{R} \Gamma_{\mathfrak{m}}$ and $H_{\mathfrak{m}^{\text {opp }}}^{m}=$ $\mathrm{h}^{m} \mathrm{R} \Gamma_{\mathfrak{m}^{\text {opp }}}$. The Matlis duality functor is denoted $(-)^{\prime}$ and defined on graded $A$-modules by $\left(M^{\prime}\right)_{p}=$ $\operatorname{Hom}_{k}\left(M_{-p}, k\right)$. Matlis duality exchanges graded $A$-left- and graded $A$-right-modules, and is exact and therefore well defined on derived categories.

\section{Background results}

Proposition 1.1. For $X$ and $Y$ in $\mathrm{D}_{\mathrm{fg}}^{\mathrm{b}}(\mathrm{Gr} A)$ there is a natural isomorphism

$$
\mathrm{RHom}_{A}\left(\mathrm{R} \Gamma_{\mathfrak{m}} X, Y\right) \cong \mathrm{RHom}_{A}(X, Y) .
$$

Proof. First observe that there are natural isomorphisms

$$
\begin{aligned}
\operatorname{RHom}_{A}\left(\mathrm{R} \Gamma_{\mathfrak{m}^{\mathrm{opp}}} A, Y\right) & \cong \mathrm{RHom}_{A}\left(\mathrm{R} \Gamma_{\mathfrak{m}^{\mathrm{opp}}} A, Y^{\prime \prime}\right) \\
& \stackrel{(\mathrm{a})}{\cong}\left(Y^{\prime} \stackrel{\mathrm{L}}{A}_{A} \mathrm{R} \Gamma_{\mathfrak{m}^{\mathrm{opp}}} A\right)^{\prime} \\
& \stackrel{(\mathrm{b})}{\cong} \mathrm{R} \Gamma_{\mathfrak{m}^{\mathrm{opp}}}\left(Y^{\prime}\right)^{\prime} \\
& \stackrel{(\mathrm{c})}{\cong} Y^{\prime \prime} \\
& \cong Y .
\end{aligned}
$$

Here (a) is by [Jor99a, Theorem 1.5] and (b) is by [Jor99a, Theorem 1.6], while (c) can be seen as follows: Let $F$ be a free resolution of $Y$ consisting of finitely generated free modules. It is then easy to see that $F^{\prime}$ is an injective resolution of $Y^{\prime}$. As $F$ consists of finitely generated free modules, $F^{\prime}$ consists of torsion graded injective modules, so $\Gamma_{\mathfrak{m}^{\text {opp }}}\left(F^{\prime}\right) \cong F^{\prime}$ whence $R \Gamma_{\mathfrak{m}^{\text {opp }}}\left(Y^{\prime}\right) \cong \Gamma_{\mathfrak{m}^{\text {opp }}}\left(F^{\prime}\right) \cong$ $F^{\prime} \cong Y^{\prime}$.

Now compute:

$$
\begin{aligned}
\operatorname{RHom}_{A}\left(\mathrm{R} \Gamma_{\mathfrak{m}} X, Y\right) & \stackrel{(\mathrm{d})}{\cong} \operatorname{RHom}_{A}\left(\mathrm{R}_{\mathfrak{m}}(A) \stackrel{\mathrm{L}}{\otimes} A, Y\right) \\
& \cong \operatorname{RHom}_{A}\left(X, \operatorname{RHom}_{A}\left(\mathrm{R} \Gamma_{\mathfrak{m}} A, Y\right)\right) \\
& \stackrel{(\mathrm{e})}{\cong} \operatorname{RHom}_{A}\left(X, \operatorname{RHom}_{A}\left(\mathrm{R} \Gamma_{\mathfrak{m}^{\text {opp }}} A, Y\right)\right) \\
& \stackrel{(\mathrm{f})}{\cong} \operatorname{RHom}_{A}(X, Y) .
\end{aligned}
$$

Here (d) is by [Jor99a, Theorem 1.6] again, while (e) is by [vdB97, Corollary 4.8] and (f) is by the previous computation.

Lemma 1.2. For $X$ in $\mathrm{D}^{-}(\mathrm{Gr} A)$ and $Y$ in $\mathrm{D}^{+}(\mathrm{Gr} A)$ there is a convergent spectral sequence

$$
E_{2}^{m n}=\operatorname{Ext}_{A}^{m}\left(\mathrm{~h}^{-n} X, Y\right) \Rightarrow \operatorname{Ext}_{A}^{m+n}(X, Y) .
$$

Proof. Let $J$ be an injective resolution of $Y$. Consider the double complex given by

$$
M^{m n}=\operatorname{Hom}_{A}\left(X^{-m}, J^{n}\right) .
$$

The spectral sequence arising from the second standard filtration of the total complex Tot $M$ gives the lemma's spectral sequence.

Lemma 1.3. For $X$ in $\mathrm{D}^{-}(\mathrm{Gr} A)$ there is a convergent spectral sequence

$$
E_{2}^{m n}=\operatorname{Tor}_{-m}^{A}\left(\mathrm{H}_{\mathfrak{m} \text { opp }}^{n} A, X\right) \Rightarrow \mathrm{H}_{\mathfrak{m}}^{m+n} X .
$$




\section{LINEAR FREE RESOLUTIONS}

Proof. Let $F$ be a flat resolution of $X$. Consider the double complex given by

$$
M^{m n}=\left(\mathrm{R} \Gamma_{\mathfrak{m}^{\text {opp }}} A\right)^{m} \otimes_{A} F^{n} .
$$

The spectral sequence arising from the second standard filtration of Tot $M$ gives the lemma's spectral sequence.

To see that the sequence has the indicated limit, one needs the computation

$$
\operatorname{Tot} M \cong\left(\mathrm{R} \Gamma_{\mathfrak{m}^{\text {opp }}} A\right) \stackrel{\mathrm{L}}{\otimes}{ }_{A} X \stackrel{(a)}{\cong}\left(\mathrm{R} \Gamma_{\mathfrak{m}} A\right) \stackrel{\mathrm{L}}{\otimes} A \stackrel{(b)}{\cong} \mathrm{R} \Gamma_{\mathfrak{m}} X,
$$

where (a) is by [vdB97, Corollary 4.8] and (b) is by [Jor99a, Theorem 1.6].

\section{Two notions of regularity}

The following is almost the classical definition of Castelnuovo-Mumford regularity of graded modules, given over polynomial algebras in [EG84, Definition, p. 95] and more generally in [Jor99b, Definition 2.1].

Definition 2.1 (Castelnuovo-Mumford regularity). The complex $X$ in $\mathrm{D}(\mathrm{Gr} A)$ is called $p$-regular if

for all $m$.

$$
\mathrm{H}_{\mathfrak{m}}^{m}(X) \geqslant p+1-m=0
$$

If $X$ is $p$-regular but not $(p-1)$-regular, then I define the Castelnuovo-Mumford regularity of $X$ to be

$$
\text { CMreg } X=p \text {. }
$$

If $X$ is not $p$-regular for any $p$, then CMreg $X=\infty$. If $X$ is $p$-regular for every $p$ (that is, if $\mathrm{H}_{\mathfrak{m}}(X)=0$ ), then CMreg $X=-\infty$.

The following is the competing definition of Castelnuovo-Mumford regularity given in [AE92]. In order not to confuse things, I have to use a different name.

Definition 2.2 (Ext-regularity). The complex $X$ in $\mathrm{D}(\mathrm{Gr} A)$ is called $r$-Ext-regular if

$$
\operatorname{Ext}_{A}^{m}(X, k)_{\leqslant-r-1-m}=0
$$

for all $m$.

If $X$ is $r$-Ext-regular but not $(r-1)$-Ext-regular, then I define the Ext-regularity of $X$ to be

$$
\text { Ext.reg } X=r \text {. }
$$

If $X$ is not $r$-Ext-regular for any $r$, then Ext.reg $X=\infty$. If $X$ is $r$-Ext-regular for every $r$ (that is, if $\left.\operatorname{Ext}_{A}(X, k)=0\right)$, then Ext.reg $X=-\infty$.

Observation 2.3. Let $X$ in $\mathrm{D}_{\mathrm{fg}}^{\mathrm{b}}(\mathrm{Gr} A)$ have $X \neq 0$. Since $A$ has a balanced dualizing complex, the local duality theorem [Yek92, Theorem 4.18] holds, so $\mathrm{R} \Gamma_{\mathfrak{m}}(X)^{\prime}$ is in $\mathrm{D}_{\mathrm{fg}}^{\mathrm{b}}\left(\mathrm{Gr} A^{\mathrm{opp}}\right)$ and has $\mathrm{R} \Gamma_{\mathfrak{m}}(X)^{\prime} \not 0$. Hence CMreg $X \neq \pm \infty$.

By [vdB97, Corollary 4.8] I have $\mathrm{H}_{\mathfrak{m}}^{n} A \cong \mathrm{H}_{\mathfrak{m}}^{n}$ opp $A$ for each $n$, whence

$$
\operatorname{CMreg}\left({ }_{A} A\right)=\operatorname{CMreg}\left(A_{A}\right) \text {. }
$$

I denote this number by CMreg $A$.

Observation 2.4. Let $X$ in $\mathrm{D}_{\mathrm{fg}}^{\mathrm{b}}(\operatorname{Gr} A)$ have $X \neq 0$. It is easy to see that $\operatorname{Ext}_{A}(X, k) \neq 0$ whence Ext.reg $X \neq-\infty$. However, Ext.reg $X=\infty$ is possible. 


\section{P. JøRGENSEN}

If $F$ is a minimal free resolution of $X$, then $X$ is $r$-Ext-regular exactly if the generators of $F_{m}$ are placed in degrees less than or equal to $r+m$ for each $m$. This has a nice consequence: From considering $\operatorname{Tor}^{A}\left(k_{A},{ }_{A} k\right)$ it follows that the minimal free resolutions of $k_{A}$ and ${ }_{A} k$ have their generators placed in the same degrees. Hence

$$
\operatorname{Ext} \cdot \operatorname{reg}\left(k_{A}\right)=\operatorname{Ext} \cdot \operatorname{reg}\left({ }_{A} k\right) .
$$

I denote this number by Ext.reg $k$.

The following two theorems show that the notions of Castelnuovo-Mumford and Ext-regularity enjoy a close relationship. Note the structural similarity between the proofs.

Theorem 2.5. Given $X$ in $\mathrm{D}_{\mathrm{fg}}^{\mathrm{b}}(\mathrm{Gr} A)$ with $X \neq 0$. Then

$$
\text { Ext.reg } X \leqslant \text { CMreg } X+\text { Ext.reg } k \text {. }
$$

Proof. Observation 2.3 gives CMreg $X \neq-\infty$, so for Ext.reg $k=\infty$ the theorem makes sense and holds trivially. So I can assume that Ext.reg $k=r$ is finite. By Observation 2.4, the minimal free resolution $F$ of $k_{A}$ then has the generators of $F_{m}$ placed in degrees less than or equal to $r+m$ for each $m$, so $F_{m}$ can be written as a finite coproduct

$$
F_{m}=\coprod_{j} A\left(-\sigma_{m j}\right)
$$

with $\sigma_{m j} \leqslant r+m$. Taking Matlis duals, $I=F^{\prime}$ is a minimal injective resolution of ${ }_{A} k$ which has

$$
I^{m}=\coprod_{j} A^{\prime}\left(\sigma_{m j}\right)
$$

still with

$$
\sigma_{m j} \leqslant r+m .
$$

Set $p=\operatorname{CMreg} X$ and $Z=\mathrm{R}_{\mathfrak{m}} X$. Then

$$
\mathrm{h}^{-n}(Z)_{\geqslant p+1+n}=\mathrm{h}^{-n}\left(\mathrm{R} \Gamma_{\mathfrak{m}} X\right)_{\geqslant p+1+n}=\mathrm{H}_{\mathfrak{m}}^{-n}(X)_{\geqslant p+1+n}=0
$$

for each $n$, whence

$$
\left(\left(\mathrm{h}^{-n} Z\right)^{\prime}\right) \leqslant-p-1-n=0 .
$$

Now, $\operatorname{Ext}_{A}^{m}\left(\mathrm{~h}^{-n} Z, k\right)$ is a subquotient of

$$
\operatorname{Hom}_{A}\left(\mathrm{~h}^{-n} Z, I^{m}\right)=\operatorname{Hom}_{A}\left(\mathrm{~h}^{-n} Z, \coprod_{j} A^{\prime}\left(\sigma_{m j}\right)\right) \cong \coprod_{j}\left(\mathrm{~h}^{-n} Z\right)^{\prime}\left(\sigma_{m j}\right),
$$

and this vanishes in degrees less than or equal to $-p-1-n-r-m$ by Equations (1) and (2), so also

$$
\operatorname{Ext}_{A}^{m}\left(\mathrm{~h}^{-n} Z, k\right)_{\leqslant-p-1-n-r-m}=0 .
$$

Lemma 1.2 provides a convergent spectral sequence

$$
E_{2}^{m n}=\operatorname{Ext}_{A}^{m}\left(\mathrm{~h}^{-n} Z, k\right) \Rightarrow \operatorname{Ext}_{A}^{m+n}(Z, k),
$$

and since Equation $(3)$ shows $\left(E_{2}^{m n}\right)_{\leqslant-p-1-r-(m+n)}=0$ it follows that

$$
\operatorname{Ext}_{A}^{q}(Z, k)_{\leqslant-p-1-r-q}=0
$$

for each $q$.

Finally, Proposition 1.1 gives

$$
\begin{gathered}
\operatorname{Ext}_{A}^{q}(Z, k)=\operatorname{Ext}_{A}^{q}\left(\mathrm{R} \Gamma_{\mathfrak{m}} X, k\right) \cong \operatorname{Ext}_{A}^{q}(X, k), \\
1056
\end{gathered}
$$




\section{LINEAR FREE RESOLUTIONS}

so Equation (4) implies

$$
\operatorname{Ext}_{A}^{q}(X, k)_{\leqslant-p-1-r-q}=0
$$

for each $q$, showing

$$
\text { Ext.reg } X \leqslant p+r=\text { CMreg } X+\text { Ext.reg } k \text {. }
$$

Theorem 2.6. Given $X$ in $\mathrm{D}_{\mathrm{fg}}^{\mathrm{b}}(\mathrm{Gr} A)$ with $X \neq 0$. Then

$$
\text { CMreg } X \leqslant \text { Ext.reg } X+\operatorname{CMreg} A \text {. }
$$

Proof. Observation 2.3 gives CMreg $A \neq-\infty$, so for Ext.reg $X=\infty$ the theorem makes sense and holds trivially. So I can assume that Ext.reg $X=r$ is finite. By Observation 2.4, the minimal free resolution $F$ of $X$ then has the generators of $F_{m}$ placed in degrees less than or equal to $r+m$ for each $m$, so $F_{m}$ can be written as a finite coproduct

$$
F_{m}=\coprod_{j} A\left(-\sigma_{m j}\right)
$$

with

$$
\sigma_{m j} \leqslant r+m .
$$

Set $p=\operatorname{CMreg} A$. Observation 2.3 gives $\operatorname{CMreg}\left(A_{A}\right)=\operatorname{CMreg} A$, so I get

$$
\mathrm{H}_{\mathfrak{m}^{\text {opp }}}^{n}(A)_{\geqslant p+1-n}=0
$$

for each $n$.

Now, $\operatorname{Tor}_{-m}^{A}\left(\mathrm{H}_{\mathfrak{m}^{\text {opp }}}^{n} A, X\right)$ is a subquotient of

$$
\mathrm{H}_{\mathfrak{m}^{\text {opp }}}^{n}(A) \otimes_{A} F_{-m} \cong \mathrm{H}_{\mathfrak{m}^{\text {opp }}}^{n}(A) \otimes_{A} \coprod_{j} A\left(-\sigma_{-m, j}\right) \cong \coprod_{j} \mathrm{H}_{\mathfrak{m}^{\text {opp }}}^{n}(A)\left(-\sigma_{-m, j}\right),
$$

and this vanishes in degrees larger than or equal to $p+1-n+r-m$ by Equations (5) and (6), so also

$$
\operatorname{Tor}_{-m}^{A}\left(\mathrm{H}_{\mathfrak{m} \text { opp }}^{n} A, X\right)_{\geqslant p+1-n+r-m}=0 .
$$

Lemma 1.3 provides a convergent spectral sequence

$$
E_{2}^{m n}=\operatorname{Tor}_{-m}^{A}\left(\mathrm{H}_{\mathfrak{m}}^{n} \text { opp } A, X\right) \Rightarrow \mathrm{H}_{\mathfrak{m}}^{m+n} X
$$

and since Equation $(7)$ shows $\left(E_{2}^{m n}\right)_{\geqslant p+1+r-(m+n)}=0$, it follows that

$$
\mathrm{H}_{\mathfrak{m}}^{q}(X)_{\geqslant p+1+r-q}=0
$$

for each $q$, showing

$$
\text { CMreg } X \leqslant p+r=\text { Ext.reg } X+\operatorname{CMreg} A \text {. }
$$

Let me end the section with some easy consequences. First recall the following definition.

Definition 2.7. The algebra $A$ is called Koszul if Ext.reg $k=0$.

For $A$ to be Koszul means exactly that the minimal free resolutions of ${ }_{A} k$ and $k_{A}$ are linear; cf. Observation 2.4.

The following corollary is immediate from Theorems 2.5 and 2.6.

Corollary 2.8. Suppose that $A$ is Koszul and has CMreg $A=0$. Then each $X$ in $\mathrm{D}_{\mathrm{fg}}^{\mathrm{b}}(\mathrm{Gr} A)$ has Ext.reg $X=$ CMreg $X$.

The following corollary is also immediate from Observation 2.3 and Theorem 2.5. It extends [AE92, Theorem 1] and [AP01, Theorem 1] to the non-commutative case.

Corollary 2.9. Suppose that $A$ has Ext.reg $k<\infty$. Then each $X$ in $\mathrm{D}_{\mathrm{fg}}^{\mathrm{b}}(\mathrm{Gr} A)$ has Ext.reg $X<\infty$. 


\section{LINEAR FREE RESOLUTIONS}

\section{Linear free resolutions}

The following main result is a simultaneous extension of [AE92, Corollary 2] (to the non-commutative case) and [Jor99b, Theorem 2.6] (to the non-AS-regular case).

Recall that $A$ is the algebra of Setup 0.1.

Theorem 3.1. Suppose that $A$ is Koszul, and let $M$ in $\mathrm{Gr} A$ be finitely generated with $M \neq 0$. Then for $s \geqslant \operatorname{CMreg} M$, the minimal free resolution of $M_{\geqslant s}(s)$ is linear. (Note that CMreg $M$ is finite.)

Proof. The result clearly holds if $M_{\geqslant s}(s)$ is 0 , so I can assume $M_{\geqslant s}(s) \nRightarrow 0$.

Let $F$ be the minimal free resolution of $M_{\geqslant s}(s)$. As $M_{\geqslant s}(s)$ sits in non-negative degrees, it is clear for each $m$ that $F_{m}$ has no generators placed in degrees strictly smaller than $m$. Hence it is enough to prove for each $m$ that $F_{m}$ also has no generators placed in degrees strictly larger than $m$. By Observation 2.4 this is the same as proving

$$
\operatorname{Ext} \cdot \operatorname{reg}\left(M_{\geqslant s}(s)\right) \leqslant 0 .
$$

Since $A$ is Koszul, Ext.reg $k=0$ holds. By Theorem 2.5, the inequality (9) will therefore follow from $\operatorname{CMreg}\left(M_{\geqslant s}(s)\right) \leqslant 0$, which is again the same as $\operatorname{CMreg}\left(M_{\geqslant s}\right) \leqslant s$, that is

$$
\mathrm{H}_{\mathfrak{m}}^{m}\left(M_{\geqslant s}\right)_{\geqslant s+1-m}=0
$$

for each $m$. To show this is easy: there is a short exact sequence $0 \longrightarrow M_{\geqslant s} \longrightarrow M \longrightarrow M / M_{\geqslant s} \longrightarrow 0$ resulting in a long exact sequence consisting of pieces

$$
\mathrm{H}_{\mathfrak{m}}^{m}\left(M_{\geqslant s}\right) \longrightarrow \mathrm{H}_{\mathfrak{m}}^{m}(M) \longrightarrow \mathrm{H}_{\mathfrak{m}}^{m}\left(M / M_{\geqslant s}\right) .
$$

Now combine this with $\mathrm{H}_{\mathfrak{m}}^{m}(M)_{\geqslant s+1-m}=0$ for each $m$ (because $s \geqslant$ CMreg $M$ ) and

$$
\mathrm{H}_{\mathfrak{m}}^{m}\left(M / M_{\geqslant s}\right) \cong \begin{cases}M / M_{\geqslant s} & \text { for } m=0 \\ 0 & \text { for } m \geqslant 1\end{cases}
$$

(because $M / M_{\geqslant s}$ is torsion).

\section{REFERENCES}

AE92 L. L. Avramov and D. Eisenbud, Regularity of modules over a Koszul algebra, J. Algebra 153 (1992), $85-90$.

AP01 L. L. Avramov and I. Peeva, Finite regularity and Koszul algebras, Amer. J. Math. 123 (2001), 275-281.

EG84 D. Eisenbud and S. Goto, Linear free resolutions and minimal multiplicity, J. Algebra 88 (1984), 89-133.

Jor99a P. Jørgensen, Gorenstein homomorphisms of non-commutative rings, J. Algebra 211 (1999), 240-267.

Jor99b P. Jørgensen, Non-commutative Castelnuovo-Mumford regularity, Math. Proc. Camb. Phil. Soc. 125 (1999), 203-221.

vdB97 M. van den Bergh, Existence theorems for dualizing complexes over non-commutative graded and filtered rings, J. Algebra 195 (1997), 662-679.

Yek92 A. Yekutieli, Dualizing complexes over noncommutative graded algebras, J. Algebra 153 (1992), $41-84$.

Peter Jørgensen popjoerg@maths.leeds.ac.uk

Department of Pure Mathematics, University of Leeds, Leeds LS2 9JT, UK 